The University of Southern Mississippi

The Aquila Digital Community

Faculty Publications

4-1-1997

\title{
Conformation of Interacting Polymer Chains: Effects of Temperature, Bias, Polymer Concentration, and Porosity
}

Grace M. Foo

University of Southern MIssissippi

Ras B. Pandey

University of Southern Mississippi, ras.pandey@usm.edu

Follow this and additional works at: https://aquila.usm.edu/fac_pubs

Part of the Physics Commons

\section{Recommended Citation}

Foo, G. M., Pandey, R. B. (1997). Conformation of Interacting Polymer Chains: Effects of Temperature, Bias, Polymer Concentration, and Porosity. Physical Review E, 55(4), 4433-4441.

Available at: https://aquila.usm.edu/fac_pubs/5294

This Article is brought to you for free and open access by The Aquila Digital Community. It has been accepted for inclusion in Faculty Publications by an authorized administrator of The Aquila Digital Community. For more information, please contact Joshua.Cromwell@usm.edu. 


\title{
Conformation of interacting polymer chains: Effects of temperature, bias, polymer concentration, and porosity
}

\author{
Grace M. Foo ${ }^{1,2}$ and R. B. Pandey ${ }^{1,3}$ \\ ${ }^{1}$ Program in Scientific Computing, University of Southern Mississippi, Hattiesburg, Mississippi 39406-5046 \\ ${ }^{2}$ Supercomputing and Visualization Unit, Computer Center, National University of Singapore, 119260 Singapore \\ ${ }^{3}$ Department of Physics and Astronomy, University of Southern Mississippi, Hattiesburg, Mississippi 39406-5046
}

(Received 10 May 1996; revised manuscript received 6 December 1996)

\begin{abstract}
The conformations of interacting polymer chains driven by a biased field in heterogeneous media are studied using Monte Carlo simulations in three dimensions. In addition to excluded volume, a nearest-neighbor interaction is considered with polymer-polymer repulsion and polymer-solvent attraction. Two types of heterogeneous media are considered: (i) a homogeneous-annealed system consisting of mobile polymer chains and solvents and (ii) quenched porous media, generated by adding a random distribution of quenched barriers. Effects of polymer concentration $(p)$, bias $(B)$, temperature $(T)$, and porosity $\left(p_{s}\right)$ on the magnitude of the radius of gyration $\left(R_{g}\right)$ of the chains and its scaling with the chain length $\left(L_{c}\right)$ are studied. In an annealed system, we observe a crossover in power-law variation of the radius of gyration with the chain length, $R_{g} \sim L_{c}^{\gamma}$, from an extended conformation with $\gamma \simeq 0.7$ at low bias $(B=0.2)$, low $p$, and high $T$ to a collapsed conformation with $\gamma \sim 0.20-0.31$ at high bias $(B \geqslant 0.5)$ and low $T$. In a quenched porous medium, we observe a somewhat lower value of the power-law exponent, $\gamma \sim 0.60-0.70$, from its annealed value at high $T$ and low bias. At low temperatures, in contrast, the magnitude of $\gamma \sim 0.39-0.47$ is enhanced with respect to its annealed value. Various nonlinear responses of $R_{g}$ to bias are observed in different ranges of $B, L_{c}, p_{s}$, and $T$. In particular, we find that the response is nonmonotonic at low temperatures $(T \simeq 0.1)$ in the annealed system and at high temperatures $(T \simeq 100.0)$ in a porous medium with a relatively high barrier concentration $\left(p_{b} \geqslant 0.3\right)$. [S1063-651X(97)08804-1]
\end{abstract}

PACS number(s): 36.20.Ey, 83.10.Nn, 02.70.Lq

\section{INTRODUCTION}

Because of its fundamental and practical importance, the conformational and dynamical properties of chain polymers have attracted a considerable interest in recent years [1-5]. The conformational and transport properties of the model systems of neutral polymer chains with the excluded volume interaction have been extensively studied from a dilute solution to the melt regime. The crossover from a self-avoiding walk (SAW) conformation in the dilute regime to an ideal (random walk) configuration in the melt is well understood. There is a large variety of polymer systems [6-8] where interactions among the constituents play an important role in their conformations. Some examples include polyelectrolytes and polyampholytes (protein, DNA, and RNA to weakly charged systems with a fluctuation in the charge distribution). The long range Coulomb interactions among the constituents, on the other hand, impose a severe constraint in studying these systems both by analytical theories as well as by computer simulations. It is very difficult to perform a controlled experiment to understand the fundamental properties of the constituents of such complex systems. Computer simulation, therefore, remains one of the primary tools to investigate even simplified models of such complex systems which cannot be tackled by analytical theory in a controlled fashion. The growth in the literature in the area of computer simulation modeling of polymers is too extensive to cite all of them [5].

Several attempts have recently been made to study the problems in such complex systems via models [5-10]. Most models resort to simplified systems with a restricted range of interactions, short chain lengths, low polymer concentrations, etc., due to difficulties in taking into account the specific type and range of interaction and polydispersity among the other variables. The simplified models are, however, useful in capturing some of the important details of such complex systems. In a highly concentrated melt where the long range interactions seem to be screened out, a short range interaction, different from the original long range interaction, may be able to capture the relevant details. Further advantages of the simplified models are the feasibility of incorporating other parameters such as temperature, solvents, and their concentrations which would have been difficult otherwise. In fact, several studies have been recently reported on such simplified models which have shown interesting properties due to the interplay between the temperature and interactions [5].

The effects of external field (i.e., bias) in such interacting systems are relatively less explored. Such studies [11-13] are nevertheless useful in understanding some of the fundamental issues in electrophoretic transport, filtering, and phase-separating processes. Studying the physical properties of even simplified model chains in a quenched porous medium is more difficult [14-21]. Inclusion of an external flow field helps in reducing the relaxation time for the motion of the chains, at least for certain values of the parameter space (i.e., high porosity, low polymer concentration, and low field) [13]. On the other hand, the biased flow field makes the problem more complex as the field begins to compete with the barriers for the chains' movement $[11,12]$. In previous 
studies, we have explored the dynamics and conformational behavior of model polymer chains in porous media, with [13] and without [21] a field, in which only the excluded volume (hard-core) interaction among the chains was considered. The polymer chains could move only by reptation dynamics into "empty" neighboring sites, i.e., those that were occupied by solvent. In the present work, a more general model is considered by introducing a local nearest-neighbor (NN) interaction with a polymer-polymer repulsion and a polymer-solvent attraction. Unlike our previous studies, we are able to investigate the effect of temperature on the conformational properties of interacting chains along with the influence of their lengths and concentrations.

We have carried out a detailed study of the conformational properties of interacting chains in a background of solvent using a simplified model described in the following section (Sec. II). The conformations of chains in a homogeneous (or annealed chains) system are discussed in Sec. III and the effects of quenched barriers in Sec. IV with a summary in Sec. V.

\section{MODEL}

The simulations are performed on a simple cubic lattice of size $L \times L \times L$. A fraction $p$ of the lattice sites is randomly occupied by $n_{c}$ polymer chains, each of length $L_{c}$ (or $L_{c}+1$ nodes $)$, where $n_{c}=p L^{d} /\left(L_{c}+1\right)$. Chains are placed on the lattice by a constrained self-avoiding walk (SAW) process, each starting from a randomly selected site. A fraction $p_{b}$ of the lattice sites, randomly selected from the empty lattice sites, is then occupied by quenched barriers. A site cannot be occupied by more than one barrier or chain node. The porosity of the medium is defined by $p_{s}=1-p_{b}$.

In addition to excluded volume, we consider nearestneighbor polymer-polymer repulsion and polymer-solvent attraction. An effective charge or interaction density $\rho$ is assigned to each lattice site, where $\rho=1$ for the polymer nodes, 0 for the barriers, and -1 for the empty sites. The interaction energy of the system is described by

$$
E=\sum_{i j} \rho_{i} \rho_{j}
$$

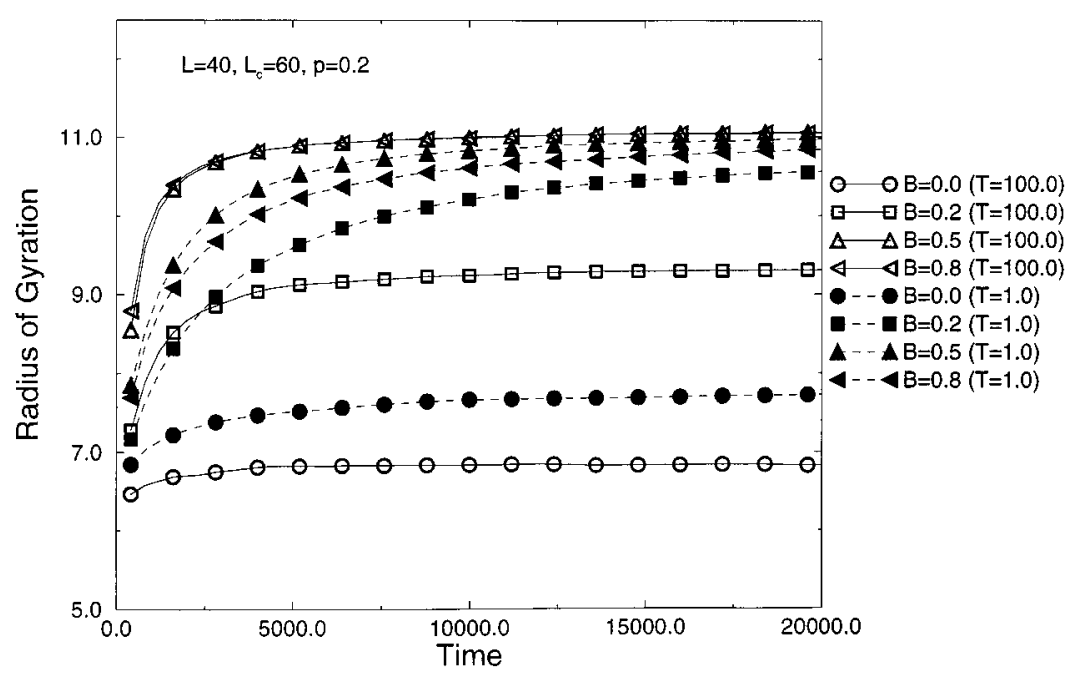

FIG. 1. Radius of gyration versus time for driven chains at concentration $p=0.2$ and length $L_{c}=60$. where the summation is restricted to nearest-neighbor sites. Note that such Ising-like effective interactions are frequently used to study a variety of issues in different contexts, i.e., simple fluids [22], polymer chains [5], etc., for simplicity. A biased field is also considered in moving the nodes as the chains explore their conformational phase space via kink jump and reptation.

The chain nodes are selected randomly. If the node is one of the end nodes (head or tail) of the chain, then we attempt to reptate the chain with the following rule: We select one of its neighboring lattice sites $j$ with probability $B$ in the direction of the field and in any direction with probability $1-B$, where $B(0 \leqslant B \leqslant 1)$ is the biased probability. If the neighboring site is empty, then we attempt to reptate the chain with a Boltzmann distribution using a Monte Carlo acceptance condition

$$
r \leqslant \exp (-\Delta E / T)
$$

where $r$ is a random number $(0<r<1), \Delta E$ is the difference in interaction energy between the new and old configurations, and $T$ is the temperature in units of the Boltzmann constant.

On the other hand, if the randomly selected node is one of the interior nodes of the chain, then a kink-jump move is attempted. In this case, one of the nearest- or next-nearestneighbor sites $j$ is selected with the biased probability described above. If site $j$ is empty, then we attempt to move the node and the associated bonds via a Boltzmann distribution [Eq. (2)]. However, if the neighboring site $j$ is occupied, the move fails and the node remains in its old position. An attempt to move each node of all chains once regardless of its success is defined as a Monte Carlo Step (MCS). A periodic boundary condition is used for the moves across the sample. The chains are moved for a fixed (large) number of time steps, during which we monitor various physical quantities.

The parameters are the concentration $p$ of the chains, chain length $L_{c}$, temperature $T$, strength of external bias $B$, and concentration of barriers $p_{b}$ (or porosity $\left.p_{s}=1-p_{b}\right)$. For each set of parameters, we use as many independent simulations $n_{s}$ as permitted by the available resources to obtain a reliable estimate of the physical quantities. Chains of length $L_{c}=20,40$, and 60 were used over

$$
L_{c}=60 .
$$


external biases $B=0.0-0.8$ at polymer concentrations $p=0.1,0.2,0.3$ in the annealed system $\left(p_{b}=0.0\right)$ and at a fixed polymer concentration $p=0.3$ in the porous medium $\left(0.0<p_{b} \leqslant 0.4\right)$. Each simulation was run for a sufficiently long time to relax the chain conformations. We evaluate the radius of gyration of the chains which is defined as

$$
\begin{gathered}
R_{g}=\frac{1}{n_{s} n_{c}\left(L_{c}+1\right)} \sum_{j=1}^{n_{c}} \sum_{i=1}^{L_{c}+1} \sqrt{\left\langle\left[r_{i}(j)-r_{\mathrm{c} . \mathrm{m} .}(j)\right]^{2}\right\rangle} \\
r_{\mathrm{c} . \mathrm{m} .}=\frac{1}{L_{c}+1} \sum_{i=1}^{L_{c}+1} r_{i},
\end{gathered}
$$

where $r_{i}(j)$ is the position of the $i$ th node of the $j$ th chain; $\langle\cdots\rangle$ shows the ensemble averaging over configurational states (i.e., over MCS time).

\section{HOMOGENEOUS-ANNEALED SYSTEM}

To simplify the analysis, we first examine the behavior of the chains in a homogeneous system, i.e., in the absence of quenched barriers $\left(p_{b}=0.0\right)$. A variation of the radius of gyration with time is shown in Fig. 1 at various values of bias $B$ and two (high and low) temperatures. We immediately note that the initial conformations of the chains $(t \rightarrow 0)$ are compact (i.e., of a constrained SAW configuration) and elongate or stretch out as the chains begin to explore their conformations in the external field. The magnitude of the radius of gyration seems to approach its steady state (i.e., a nearly constant) value $\left(R_{g_{\infty}}\right)$ at almost all values of the flow field and temperatures. We define a configurational relaxation time in which the magnitude of $R_{g}$ approaches its steady state value. Our simulations show that the longer the chain, the longer it takes to reach this steady state value. At a fixed bias, the relaxation time at high temperature $(T=100.0)$ is lower than that at the low temperature ( $T=1.0)$. We further note that the magnitude of $R_{g}$ depends strongly on $B$ and that the change in $R_{g}$, i.e., the response of $R_{g}$ to bias, is larger at higher $B$.

The conformational relaxation of chains also depends on the polymer concentration, with more severe effects at low temperature (Fig. 2). As the polymer concentration increases, the relaxation time of the chains increases quite drastically at each value of the bias. For example, at bias $B=0.5$, $R_{g} \rightarrow\left(R_{g}\right)_{\infty}$ at $\sim 5.0 \times 10^{3}$ MCS for chains at $p=0.1$, while $R_{g} \rightarrow\left(R_{g}\right)_{\infty}$ at $t>2.0 \times 10^{4}$ MCS for chains at $p=0.3$. At low polymer concentration, the probability for a chain to encounter other chains during its movement is low. As the concentration increases, there is a greater probability of encountering neighboring chains. The effective percolation threshold, the concentration at which the chains begin to interfere with each other, depends on the chain length [23]. For $L_{c}=40$, this threshold should be much smaller than 0.312 [24,25], the percolation threshold for site percolation in three dimensions. Therefore, at such a high concentration as $p=0.3$, the surrounding chains begin to act like barriers. Since the field drives the chain, it begins to compete with the surrounding chains with a resulting increase in relaxation time.

\section{A. Scaling of $\boldsymbol{R}_{g}$ with chain length}

In general, for a single chain or chains in solution (from dilute to melt regime), a frequently asked question is how the

\begin{tabular}{|c|c|c|c|c|c|}
\hline \multirow{2}{*}{$\begin{array}{l}\text { Polymer conc } \\
\qquad p\end{array}$} & \multirow{2}{*}{$\begin{array}{c}\text { Barrier conc } \\
p_{b} \\
\end{array}$} & \multicolumn{4}{|c|}{ Temperature } \\
\hline & & $T=100.0$ & $T=10.0$ & $T=1.0$ & $T=0.1$ \\
\hline \multicolumn{6}{|l|}{$(B=0.2)$} \\
\hline 0.1 & 0.0 & 0.71 & 0.71 & 0.71 & 0.70 \\
\hline 0.2 & 0.0 & 0.71 & 0.71 & 0.71 & 0.74 \\
\hline 0.3 & 0.0 & 0.71 & 0.73 & & \\
\hline 0.3 & 0.1 & 0.70 & 0.70 & 0.39 & 0.45 \\
\hline 0.3 & 0.2 & 0.67 & 0.67 & 0.45 & 0.47 \\
\hline 0.3 & 0.3 & 0.68 & 0.68 & 0.44 & 0.47 \\
\hline 0.3 & 0.4 & 0.62 & 0.60 & 0.46 & 0.46 \\
\hline \multicolumn{6}{|l|}{$(B=0.5)$} \\
\hline 0.0 & 0.0 & & & & $0.31(0.29)$ \\
\hline 0.3 & 0.1 & & & $0.33(0.33)$ & $0.45(0.47)$ \\
\hline 0.3 & 0.2 & & & $0.38(0.39)$ & $0.47(0.46)$ \\
\hline 0.3 & 0.3 & & & $0.40(0.42)$ & $0.48(0.45)$ \\
\hline \multicolumn{6}{|l|}{$(B=0.8)$} \\
\hline 0.0 & 0.0 & & & & 0.20 \\
\hline 0.3 & 0.1 & & & & $0.43(0.45)$ \\
\hline 0.3 & 0.2 & & & $0.30(0.32)$ & $0.48(0.47)$ \\
\hline 0.3 & 0.3 & & & $0.40(0.40)$ & $0.48(0.48)$ \\
\hline
\end{tabular}

TABLE I. Exponent $\gamma$ in $R_{g} \sim L_{c}^{\gamma}$ for driven chains in porous media. 


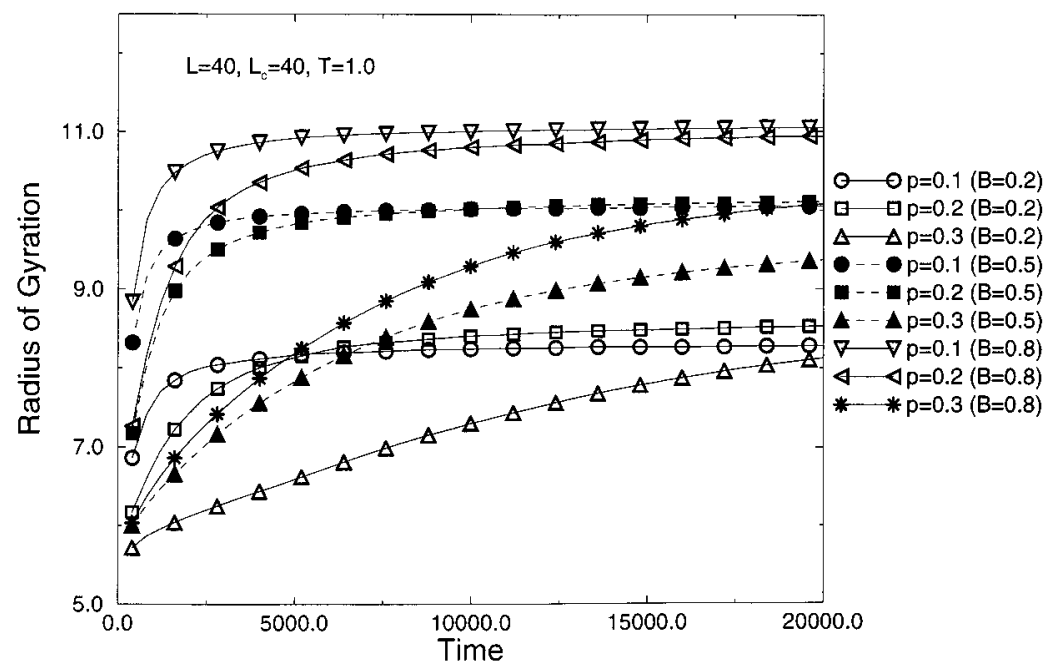

FIG. 2. Radius of gyration versus time for driven chains of length $L_{c}=40$ and temperature $T=1.0$. radius of gyration depends on the chain length $\left(L_{c}\right)$. This question is now addressed to our interacting chains driven by a field. We observe a power-law dependence of the radius of gyration on the chain length, $R_{g} \sim L^{\gamma}$, over various ranges of field, and chain and barrier concentrations. Such power-law dependence is observed at low bias $(B \leqslant 0.2)$ and concentrations $p \leqslant 0.2$ at various temperatures $0.1<T<100.0$ (Table
I). The power-law exponent $\gamma \sim 0.70-0.74$ shows little variation with temperature. At higher polymer concentration $(p=0.3)$, a power-law dependence is observed only at high temperature $(T \geqslant 10.0)$, with $\gamma \sim 0.71-0.73$ [Fig. 3(a)]. No power-law dependence is observed at high temperature and higher biases $(B \geqslant 0.5)$. At very low temperature $(T=0.1)$, high concentration $(p=0.3)$, and high bias $(B \geqslant 0.5)$, we (a)

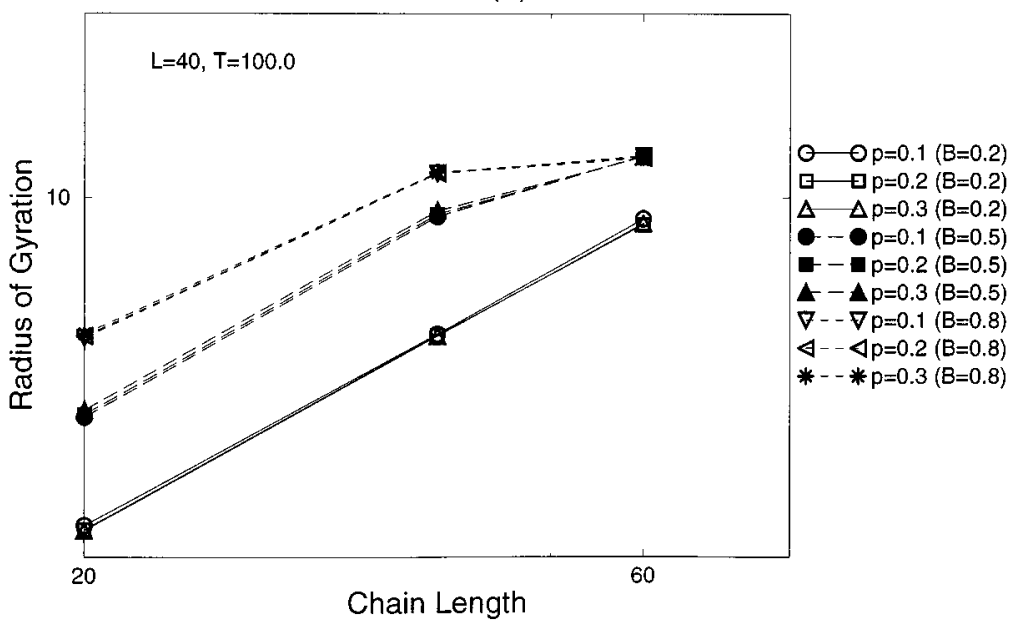

(b)

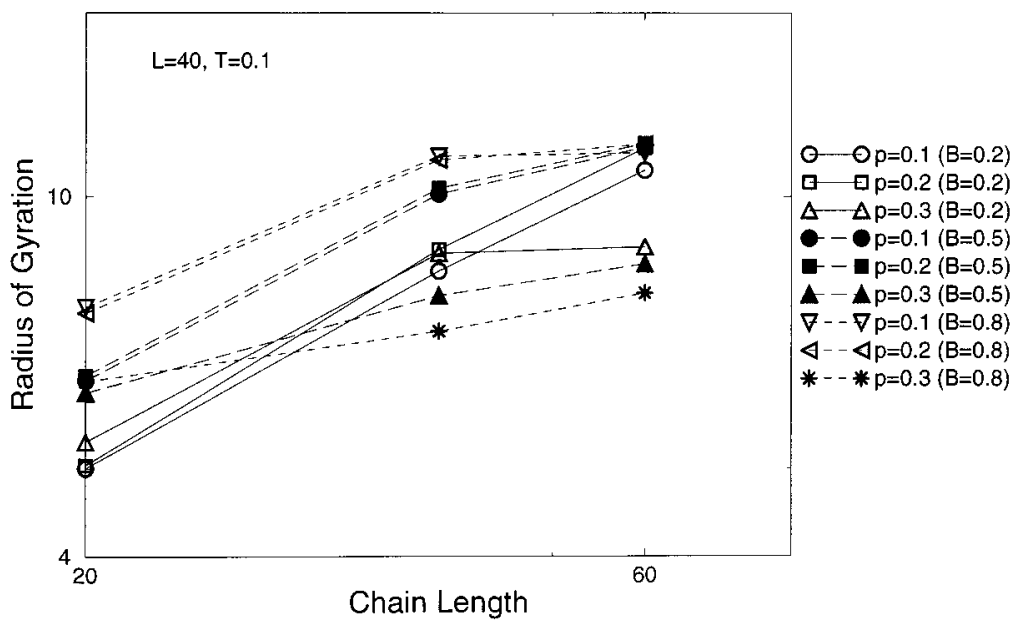

FIG. 3. Log-log plot of radius of gyration versus chain length for driven chains at concentration $p=0.3$ and (a) $T=100.0$ and (b) $T=0.1$. 
(a)

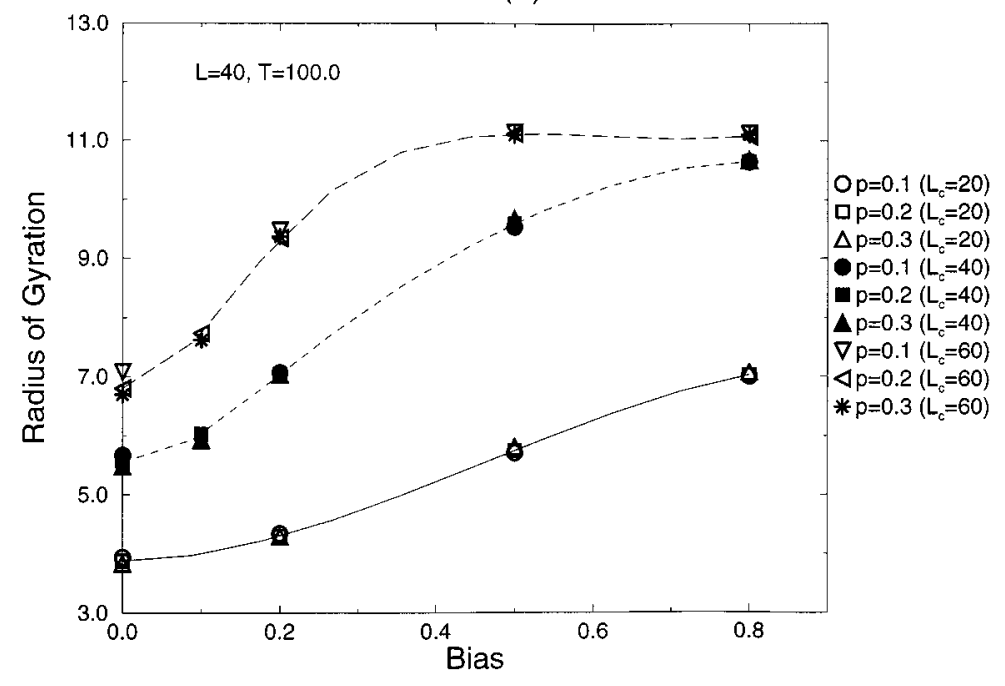

(b)

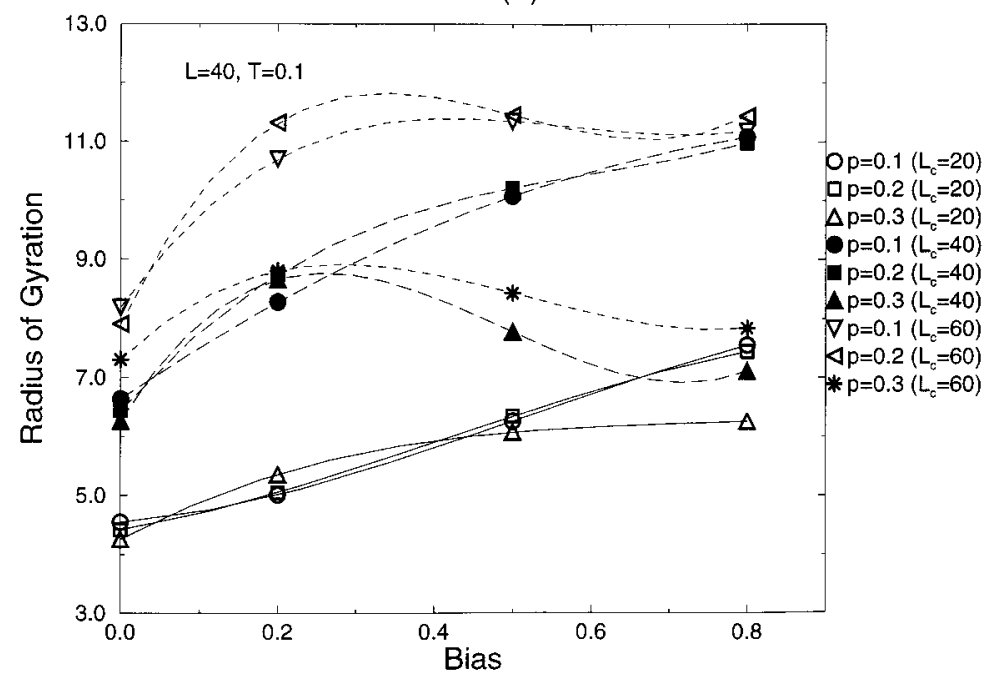

FIG. 4. Radius of gyration versus bias for chains of various lengths and concentrations, at temperature (a) $T=100.0$ and (b) $T=0.1$. seem to observe a power-law dependence on chain length with a much smaller exponent, $\gamma \sim 0.20-0.31$, which suggests the possibility of a collapsed state [Fig. 3(b)].

\section{B. Response to bias}

The response of the chain radius to the bias depends considerably on polymer concentration, chain length, and temperature. At high temperature $(T=100.0)$, we find that the rate of increase of $R_{g}$ is large at low bias followed by a decreasing rate leading to saturation of $R_{g}$ for longer chains $\left(L_{c} \geqslant 40\right)-$ a nonlinear response [Fig. 4(a)]. This qualitative response behavior remains nearly the same even on lowering the temperature to $T=1.0$, with no response to the field at $B \geqslant 0.2$ for long chains; i.e., the radius of the $L_{c}=60$ chains rapidly approaches an asymptotic limit at a rather low value of bias, $B \sim 0.2$. The short chains $\left(L_{c}=20\right)$ show nearly a linear response. Thus, we see that the chains stretch out with bias until a characteristic value, beyond which the radius saturates. This characteristic bias, as we expect, depends on the chain length and concentration, since the probability of intercepting the surrounding chains increases with the chain length and concentration. Unfortunately, we are unable to quantify this dependence due to limitations in our data points. On further reducing the temperature to $T=0.1$ [Fig. $4(\mathrm{~b})$ ], we observe that the response of the radius becomes negative beyond a certain bias $(B \geqslant 0.2)$ at high concentration $(p=0.3)$ especially for the longer chain $\left(L_{c} \geqslant 40\right)$. Competition between bias and chain obstacles leads to metastable (pinned) conformations which may be reflected by the nonmonotonic dependence.

\section{POROUS MEDIUM}

Inclusion of quenched barriers $\left(p_{b}>0\right)$ in the above system has severe effects on the relaxation of the chains and their equilibrium values at low temperature. We observe no effect of the bias on the relaxation of the radius of gyration as the chains are unable to move against barriers, even at fairly low barrier concentrations $\left(p_{b}=0.2\right)$ at low temperatures. At high temperatures $(T=100.0)$, the relaxation time increases on increasing the barrier concentration. For example, for $L_{c}=40$ chains at $T=100.0$ and bias $B=0.5$, $R_{g} \rightarrow\left(R_{g}\right)_{\infty}$ at $\sim 7.5 \times 10^{3} \mathrm{MCS}$ at $p_{b}=0.0, R_{g} \rightarrow\left(R_{g}\right)_{\infty}$ at $\sim 1.5 \times 10^{4} \mathrm{MCS}$ at $p_{b}=0.2$, and $R_{g} \rightarrow\left(R_{g}\right)_{\infty}$ at $>2.0 \times 10^{4}$ MCS at $p_{b}=0.4$ (Fig. 5). The relaxation time additionally depends on the strength of the bias. At low barrier concentrations $\left(p_{b} \leqslant 0.2\right)$, the relaxation time decreases on increasing the bias. This trend continues even to high barrier con- 


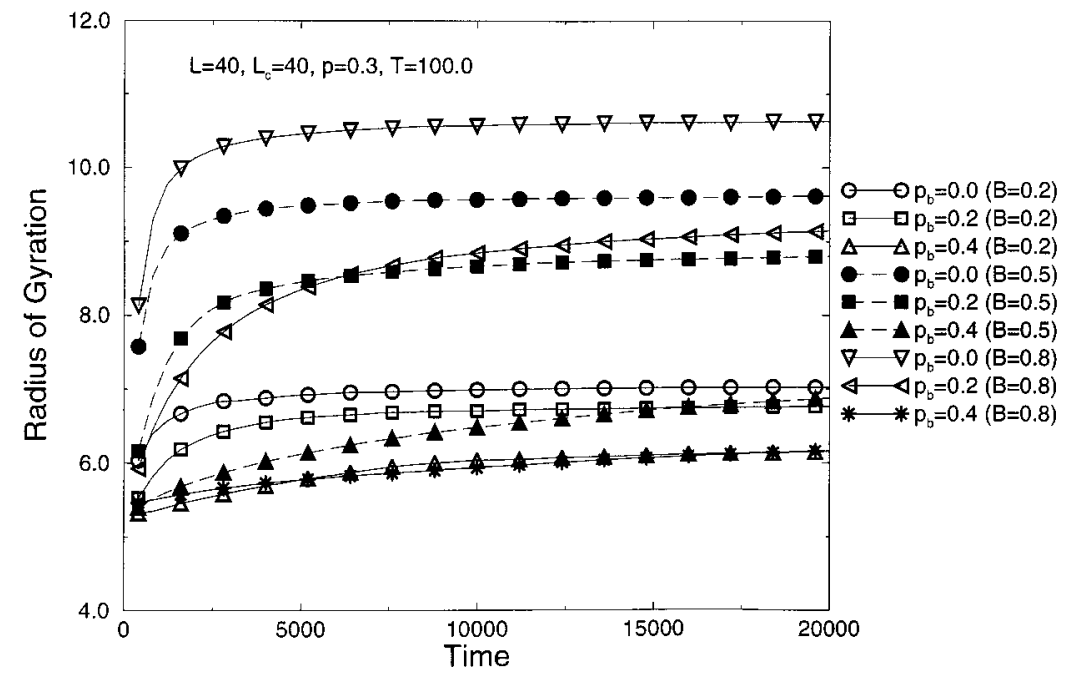

FIG. 5. Radius of gyration versus time for driven chains of length $L_{c}=40$ in porous media.

centration $\left(p_{b}=0.4\right)$, but the relaxation is affected nonmonotonically; it decreases on increasing $B$ up to a certain value, beyond which it increases due to competition between the barriers and the bias as the chains stretch out (i.e., at $\left.p_{b}=0.4, B \geqslant 0.5\right)$. (a)

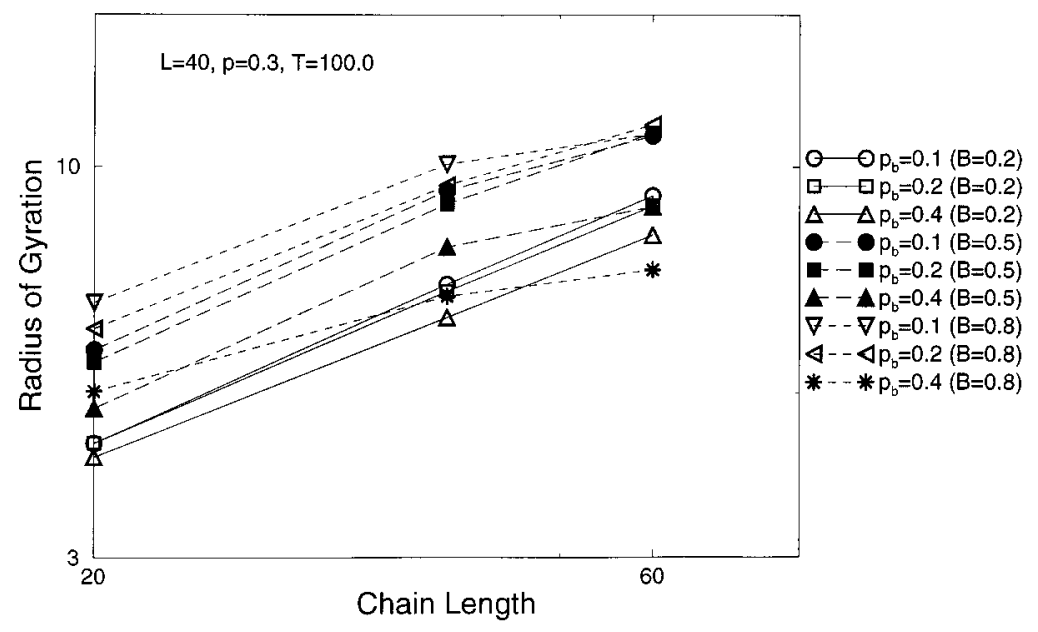

(b)

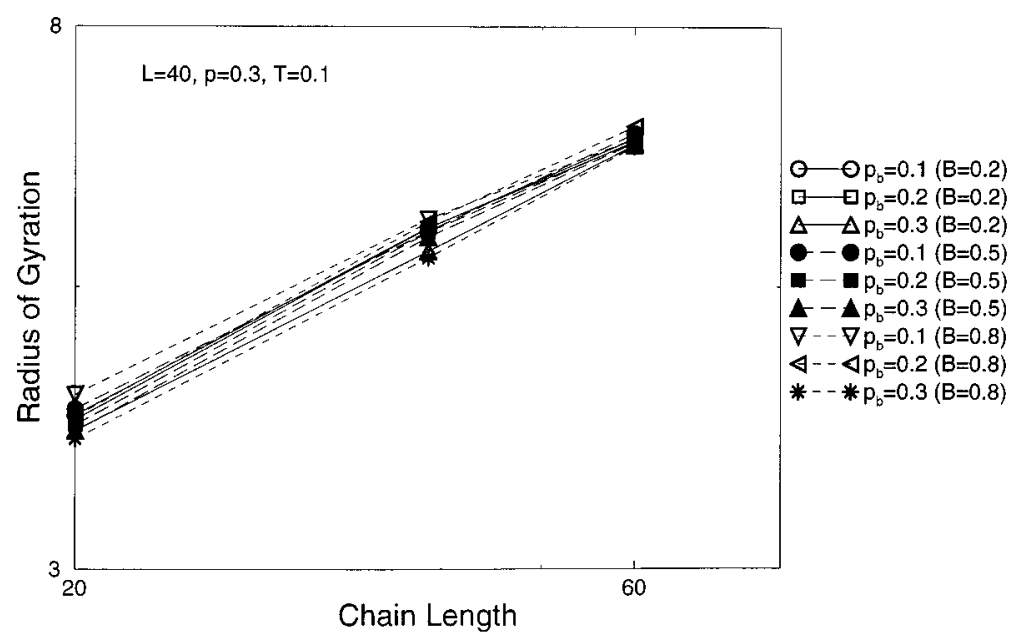

FIG. 6. Radius of gyration versus chain length for driven chains at temperature (a) $T=100.0$ and (b) $T=0.1$ in porous media. 
(a)

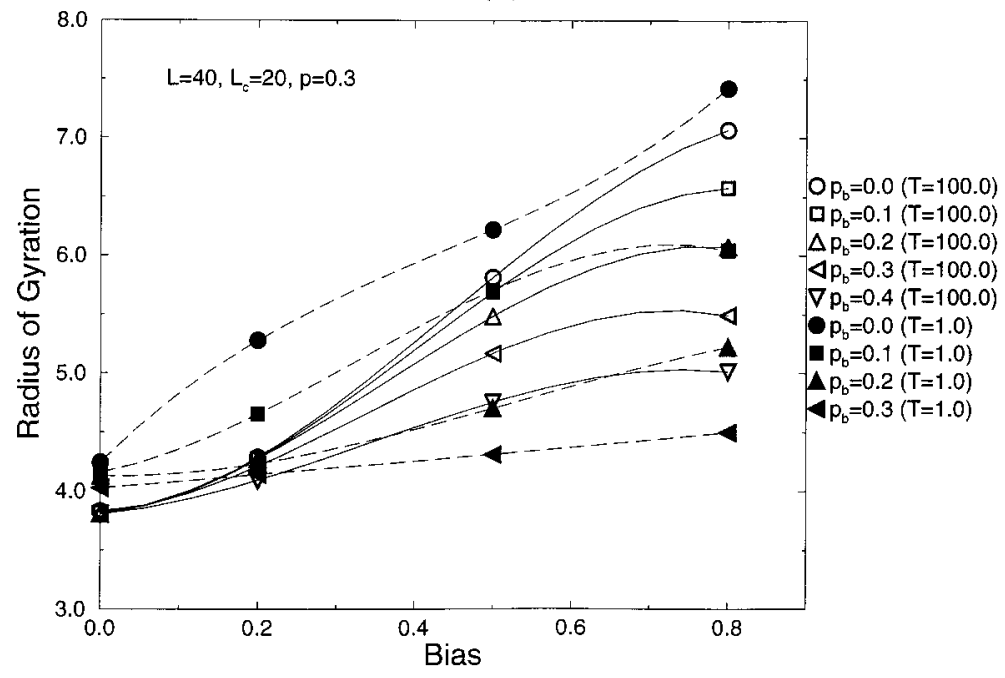

(b)

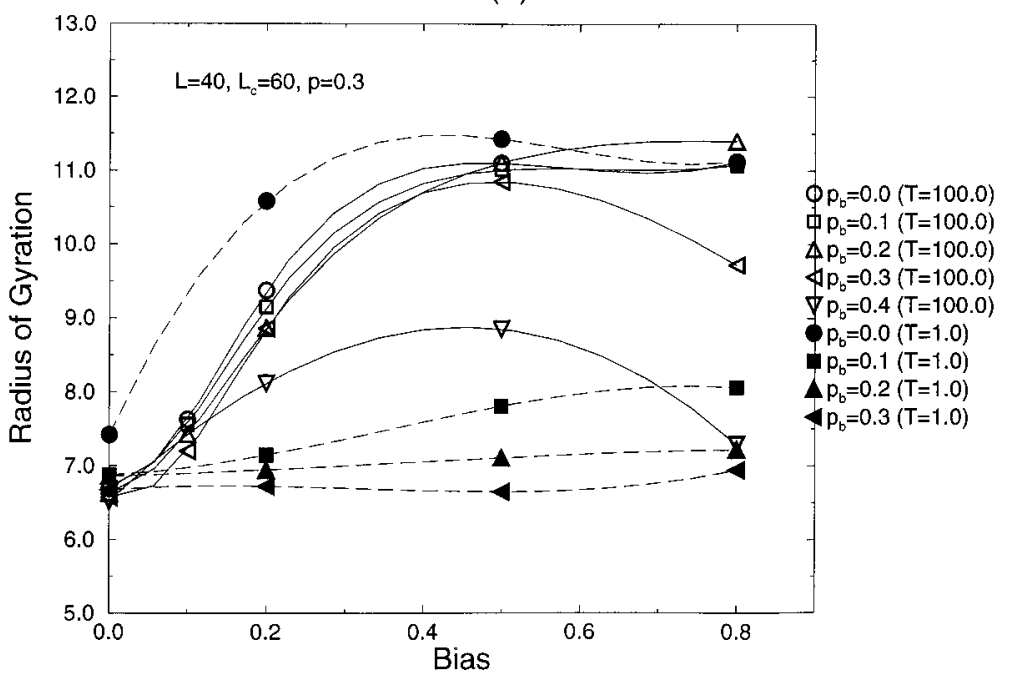

FIG. 7. Radius of gyration versus bias for chains of length (a) $L_{c}=20$ and (b) $L_{c}=60$ in porous media.
I). At high temperature $(T \geqslant 10.0)$, the exponent $\gamma \sim 0.60-0.70$ is less sensitive to barrier concentrations $p_{b} \leqslant 0.3$ [Fig. 6(a)]. But at $p_{b}=0.4, \gamma$ has dropped to $\sim 0.60-0.62$. As in the annealed case, deviations from the power-law dependence are observed at higher bias $(B \geqslant 0.5)$ and high temperature. The stretching of the chains by the field saturates at higher values of $B$ as field and barriers compete. At lower temperature $(T \leqslant 1.0), \gamma$ is not sensitive to barrier concentrations over $p_{b}=0.1-0.4$, but its magnitude has reduced considerably, i.e., $\gamma \sim 0.39-0.47$, in comparison to their values at higher temperatures [Fig. 6(b)]. At higher bias $(B \geqslant 0.5)$, the power-law dependence on chain length persists with the exponent $\gamma \sim 0.30-0.48$ (Table I) which seems to increase somewhat on increasing the barrier concentration and decreasing the temperature or bias. Note that in the absence of barriers (i.e., in a homogeneous system), the chains are collapsed while the presence of barriers seems to trap the chains in more extended configurations. Such pinning of chains due to the competition between the field and barriers becomes more pronounced on increasing the barrier concentration.

To check for finite size effects, some simulations were run on a larger-sized lattice $(L=50)$. In particular, we checked the power-law dependence at low temperature $(T \leqslant 1.0)$ and high bias $(B \geqslant 0.5)$. The power laws were recovered with estimates of the exponents (shown in parentheses) in Table I. We note some small differences with the corresponding values of exponents on the smaller lattice $\left(L_{c}=40\right)$, but these are not severe.

\section{B. Response to bias}

When barriers are introduced to the system of chains, the response of $R_{g}$ to the bias is again dependent on the chain length and temperature. For example, for short chains $\left(L_{c}=20\right)$ at concentration $p=0.3$, we find that the response of $R_{g}$ to $B$ decreases systematically on increasing the barrier concentration over the range $p_{b}=0.0-0.4$ [Fig. 7(a)]. For longer chains $\left(L_{c}=60\right)$, however, the response of the radius to $B$ is nonlinear and more sensitive to temperature [Fig. 7(b)]. At low temperature $(T=1.0)$, the presence of barriers $\left(0<p_{b} \leqslant 0.3\right)$ reduces the growth rate of $R_{g}$ substantially in comparison to those without the barriers at small values of $B$, but sustains a positive growth with increasing field. At high temperature $(T=100.0)$, however, a nonmonotonic response seems to prevail at higher values of barrier concen- 


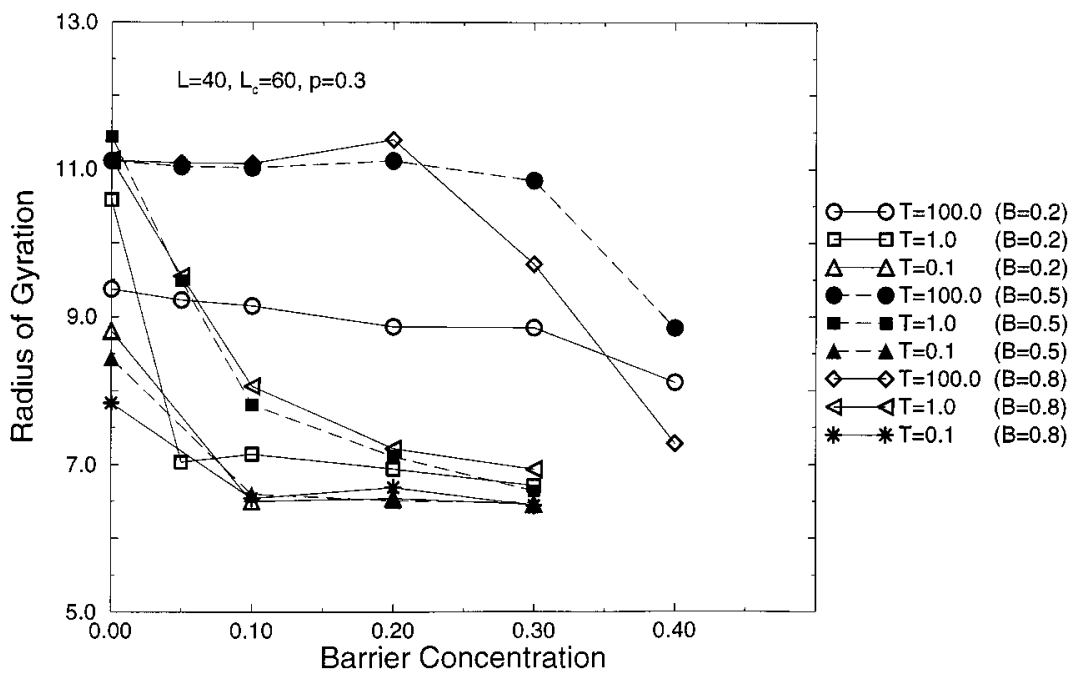

FIG. 8. Radius of gyration versus barrier concentration for driven chains of length $L_{c}=60$ and concentration $p=0.3$. tration $\left(p_{b} \geqslant 0.3\right)$. At $T=0.1$, the bias has very little effect on the chain radius when barriers are present even at low concentrations. Thus, the response of $R_{g}$ to bias is nonlinear and depends on the range of $p_{b}, T$, and $B$.

\section{Effect of barrier concentration}

A radius of gyration versus barrier concentration plot for $L_{c}=60$ chains is presented in Fig. 8. We note a significant difference in the nature of variation of $R_{g}$ with $p_{b}$ at high $(T=100.0)$ and low $(T \leqslant 1.0)$ temperatures. At high temperature, $R_{g}$ remains constant at low barrier concentrations (i.e., $p_{b}=0.0-0.3$ for $B \leqslant 0.5$ ) and drops drastically at high barrier concentrations. We note that there is a characteristic value of barrier concentration $\left(p_{b c}\right)$, above which $R_{g}$ is strongly affected by the barriers. We find that $p_{b c}$ decreases on increasing the magnitude of the bias. For example, $R_{g}$ drops significantly at $p_{b} \geqslant 0.2$ for $B=0.8$, and therefore, $p_{b c} \sim 0.2 ; \quad p_{b c} \sim 0.3$ at $B=0.5$. At low temperatures $(T \leqslant 1.0)$, since the chains are relatively less mobile, their size is restricted by the pore size, i.e., the barriers at the pore boundaries. As the barrier concentration increases, the pore size decreases; therefore the chains become more confined to the pores - this is consistent with the theoretical predictions [14-18] as well as our simulations on an athermal system of chains [21]. Thus, we see that temperature, bias, and barriers are important in governing the size of the chains.

\section{SUMMARY AND CONCLUSION}

We have used a Monte Carlo simulation to study the conformational properties of driven polymer chains in homogeneous and porous media. Interesting behaviors such as a nonlinear response and nonmonotonic dependence appear due to the competition and interplay between the external flow field, barriers, and temperature as the degree of chain entanglements is varied by varying the concentration of chains and their length.

When an external bias is applied, the chains tend to stretch in the direction of the bias. Under favorable conditions of low chain and barrier concentrations, short chain lengths, and high temperatures, the chain radius increases almost linearly with bias, leading to rodlike conformations. However, as polymer and barrier concentrations or chain lengths (mass) increase, a nonlinear response to the bias sets in with smaller responses at higher bias. This is due to the enhanced probability of intercepting the surrounding chains obstacles as the length of chains, their concentration, and the external bias increase.

We note a power-law dependence of the radius of gyration on the chain length over limited ranges of the parameters, $R_{g} \sim L_{c}^{\gamma}$. The exponent $\gamma$ at high temperature ranges from $\sim 0.70-0.74$ for chains at low chain concentrations $(p \leqslant 0.2)$ and low external bias $(B \leqslant 0.5)$ in absence of barriers $\left(p_{b}=0.0\right)$, and decreases with increasing barrier concentration, to $\sim 0.60-0.70$. At higher chain concentrations and higher bias $(B \geqslant 0.5)$ but low temperature $(T \leqslant 1.0)$, the radius also shows a power-law dependence on chain length. However, there is a sharp drop in the magnitude of the exponent $\gamma$ : In a homogeneous-annealed system, the exponent is much smaller, $\gamma \sim 0.20-0.31$, a sign of collapsed conformation, while in quenched porous medium, $\gamma \sim 0.30-0.48$ for $p_{b} \leqslant 0.3$. Thus, at low temperature, some of the chain conformations seem to get pinned by the quenched impurities. $R_{g}$ decays on increasing $p_{b}$ at low $T$ where barriers become more effective in controlling the size of the chains. At high temperatures, chain nodes attempt to move more frequently; as a result the chains are able to get out of traps and explore a larger conformational phase space. The size of the chains remains unaffected by barriers at low $p_{b}$ for $p_{b} \leqslant p_{b c}$. At $p \geqslant p_{b c}, R_{g}$ decays dramatically on increasing $p_{b}$, perhaps to collapse. $p_{b c}$ depends on $B$; the higher the field, the lower is the value of $p_{b c}$ and larger is the rate of decay of $R_{g}$.

\section{ACKNOWLEDGMENTS}

This work was supported by a NSF-EPSCoR grant. Part of the manuscript was prepared while R.B.P. was visiting the National University of Singapore. We acknowledge generous support for computer time on the CRAY YMP at the Mississippi Center for Supercomputing Research and CRAY T3D at the Pittsburgh Supercomputing Center. We thank K. Binder for useful discussion. 
[1] P. J. Flory, Principles of Polymer Chemistry (Cornell University Press, Ithaca, NY, 1953).

[2] J. D. Ferry, Viscoelsatic Properties of Polymers (Wiley, New York, 1980).

[3] P. G. de Gennes, Scaling Concepts in Polymer Physics (Cornell University Press, Ithaca, NY, 1979).

[4] M. Doi and S. F. Edwards, The Theory of Polymer Dynamics (Clarendon Press, Oxford, 1986).

[5] K. Binder, in Monte Carlo and Molecular Dynamics Simulations in Polymer Science, edited by K. Binder (Oxford University Press, New York, 1995).

[6] M. J. Stevens and K. Kremer, J. Chem. Phys. 103, 1669 (1995).

[7] B. Dunweg, M. Stevens, and K. Kremer, in Monte Carlo and Molecular Dynamics Simulations in Polymer Science [5], p. 125.

[8] Macro-ion Characterization: From Dilute Solution to Complex Fluids, edited by K. Schmitz (American Chemical Society, Washington, D.C., 1994).

[9] R. Podgornik, T. Akesson, and B. Jonsson, J. Chem. Phys. 102, 9423 (1995).

[10] M. C. Lonergan, A. Nitzan, M. A. Ratner, and D. F. Shriver, J. Chem. Phys. 103, 3253 (1995).

[11] J. Noolandi et al., Phys. Rev. Lett. 58, 2428 (1987).
[12] T. A. Kavassalis and J. Noolandi, Phys. Rev. Lett. 59, 2674 (1987).

[13] G. M. Foo and R. B. Pandey, Phys. Rev. E 51, 5738 (1995).

[14] M. Muthukumar, J. Chem. Phys. 90, 4594 (1989).

[15] A. Baumgartner and M. Muthukumar, J. Chem. Phys. 87, 3082 (1987).

[16] A. Baumgartner, Europhys. Lett. 4, 1221 (1987).

[17] M. Muthukumar and A. Baumgartner, Macromolecules 22, 1937 (1989).

[18] M. Muthukumar and A. Baumgartner, Macromolecules 22, 1941 (1989).

[19] P. Haronska and T. A. Vilgis, J. Chem. Phys. 101, 3104 (1994).

[20] F. Horkay, H. B. Stanley, E. Geissler, and S. M. King, Macromolecules 28, 678 (1995).

[21] G. M. Foo, R. B. Pandey, and D. Stauffer, Phys. Rev. E 53, 3717 (1996).

[22] Microscopic Simulation of Complex Hydrodynamic Phenomena, edited by M. Mareschal and B. L. Holian (Plenum, New York, 1992).

[23] J. Becklehimer and R. B. Pandey, J. Stat. Phys. 75, 765 (1994).

[24] D. Stauffer and A. Aharony, Introduction to Percolation Theory (Taylor \& Francis, London, 1994).

[25] M. Sahimi, Applications of Percolation Theory (Taylor \& Francis, London, 1994). 